Texas A\&M University-San Antonio

Digital Commons @ Texas A\&M University-San Antonio

2012

\title{
Statistical Analyses Cannot be Divorced From Archaeological Theory: A Reply to Potter
}

A. Mesoudi

Michael J. O'Brien

Texas A\&M University-San Antonio, Mike.Obrien@tamusa.edu

Follow this and additional works at: https://digitalcommons.tamusa.edu/hist_faculty

Part of the Anthropology Commons

\section{Repository Citation}

Mesoudi, A. and O'Brien, Michael J., "Statistical Analyses Cannot be Divorced From Archaeological Theory: A Reply to Potter" (2012). History Faculty Publications. 21.

https://digitalcommons.tamusa.edu/hist_faculty/21

This Article is brought to you for free and open access by the College of Arts and Sciences at Digital Commons @ Texas A\&M University- San Antonio. It has been accepted for inclusion in History Faculty Publications by an authorized administrator of Digital Commons @ Texas A\&M University- San Antonio. For more information, please contact deirdre.mcdonald@tamusa.edu. 


\title{
STATISTICAL ANALYSES CANNOT BE DIVORCED FROM ARCHAEOLOGICAL THEORY: A REPLY TO POTTER
}

\author{
Alex Mesoudi and Michael J. O’Brien
}

\begin{abstract}
Potter criticizes our experimental study of the roles played by indirect bias and guided variation in shaping prehistoric Great Basin projectile point variation. His criticisms are technically correct from the standpoint of statistical convention, but he fails to understand the theoretical rationale of our study. Without such an understanding, his assertion that our conclusions are questionable is incorrect. Here we point out again (1) how our experimental work bridges the gap between cultural-transmission theory and the empirical record and (2) why our conclusions are indeed valid.
\end{abstract}

Potter critica nuestro estudio experimental acerca de los papeles jugados por el sesgo indirecto y la variación guiada en la formulación de la variación de las puntas de proyectil en la Gran Cuenca. Sus críticas son técnicamente correctas desde el punto de vista de la convención estadística, pero él no entiende la justificación teórica de nuestro estudio. Sin esa comprensión, su afirmación de que nuestras conclusiones son cuestionables es incorrecta. Aquí señalamos de nuevo (l) cómo nuestro trabajo experimental conecta la laguna existente entre la teoría de transmisión cultural y los datos empíricos y (2) por qué nuestras conclusiones son, por lo tanto, válidas.

$\mathrm{W}$ e appreciate Potter's cautionary notes regarding statistical analyses reported in Mesoudi and O'Brien (2008a), specifically with respect to our use of correlations. Rigorous and appropriate statistical methods are vital for testing specific hypotheses concerning culture change, and we encourage any attempts to improve such methods. However, whereas many of Potter's statistical criticisms are technically correct with respect to statistical conventions, we take serious issue with his assertion that the validity of our conclusions is consequently questionable. Most important, he fails to acknowledge the theoretical rationale of our study. Without understanding this rationale, many of Potter's criticisms are far wide of the mark.

Our study was an experimental test of a hypothesis proposed by Bettinger and Eerkens (1999) to explain diversity in projectile points from two regions of the Great Basin of the western United States manufactured ca. A.D. 300-600, following the replacement of the atlatl with the bow and arrow. Bettinger and Eerkens observed that points from central Nevada exhibited higher correlations between attributes (length, width, thickness, and shape) than points from eastern California. They explained this regional difference in terms of cultural-transmission biases, arguing that in central Nevada point designs spread through "indirect bias" (Boyd and Richerson 1985), whereby point manufacturers copied the design of a single successful or prestigious individual in their local group. As everyone copied the same most-successful hunter, all point designs within that group tended to converge on that single hunter's design. If different groups converged on different designs, perhaps because of environmental differences or the existence of multiple locally optimal designs, then correlations across the entire region would increase. For example, if all points in group $A$ were long and thin, and all points in group B were short and wide, then across the entire region length and thickness would be correlated: long points are always thin, and short points are always wide.

Bettinger and Eerkens (1999) argued that point designs in eastern California spread not through

Alex Mesoudi a Biological and Experimental Psychology Group, School of Biological and Chemical Sciences, Queen Mary University of London, Mile End Road, London E1 4NS, United Kingdom (a.mesoudi@qmul.ac.uk)

Michael J. O'Brien $\square$ Department of Anthropology, University of Missouri, Columbia Missouri 65211. 
indirectly biased transmission but through "guided variation" (Boyd and Richerson 1985), whereby a point manufacturer acquired a design from another individual but then modified that design according to individual trial-and-error learning. If, instead of copying attributes as a complete package from a single hunter within the group, each hunter modified different attributes independently, such that each hunter converged on a different locally optimal design, then the linkage between attributes would break down and correlations between attributes across the entire region would decrease. Thus, the lower correlations in eastern California can potentially be explained by assuming that hunters engaged in guided variation and that central-Nevada hunters engaged in indirect bias.

Experimental simulations of cultural transmission offer a valuable means of testing hypotheses regarding the use and consequences of transmission biases (Mesoudi 2007). By themselves, experiments cannot definitively answer questions regarding past cultural change. But they can complement archaeological methods to provide a more complete understanding of the past: unlike archaeological methods, with experiments we can manipulate variables to test specific hypotheses, we can conduct replications to effectively "re-run" history multiple times, and we have access to complete and uninterrupted data concerning people's behavior. Our experimental simulation (Mesoudi and O'Brien 2008a) was designed to directly test Bettinger and Eerkens' (1999) aforementioned hypothesis. Groups of participants designed "virtual projectile points" for use in a simple computer "hunting" game, while we manipulated how the participants could learn. We then asked whether the transmission biases hypothesized by Bettinger and Eerkens (1999) to have generated their archaeological data-guided variation and indirect bias-generated similar patterns of variation in our experimental data.

Phase 1 of the experiment involved participants copying the designs of a pre-test group of participants after receiving information about those pretest participants' hunting scores (potentially allowing indirect bias). Phase 2 saw participants using individual trial and error to independently modify their point designs over a series of successive hunts (simulating guided variation). Phase 3 al- lowed participants to copy other members of their group (again potentially allowing indirect bias). We assumed throughout each phase that different groups of participants were learning in one of three alternative "environments" (optimal point designs) and that within each environment there were eight different locally optimal designs. As predicted, interattribute correlations were higher in Phases 1 and 3 , which simulated indirect bias, than in Phase 2, which simulated guided variation. These results supported Bettinger and Eerkens' (1999) hypothesis that higher correlations in central Nevada than in eastern California are attributable to these different learning processes.

Potter criticizes our study for (1) applying Spearman's test for correlation to non-independent data in Phase 1, when the majority of participants copied the same most-successful model, and (2) applying Spearman's test for correlation to pooled data across the three "environments" when comparing phases 2 and 3 (a third criticism is essentially the same as the second - the issue of stratified data). Whereas Potter's points are technically and conventionally correct-that Spearman's correlation test requires independent data points and nonstratified populations - our use of these tests are nevertheless useful given the theoretical rationale outlined above. Indeed, what Potter labels as "spurious" correlations are exactly what we predicted-that they are indicative of the specific learning processes for which we were testing.

Regarding Potter's first criticism, although he is correct that the point designs in Phase 1 are not independent because they were all copied from the same pretest model, this is exactly what is predicted by Bettinger and Eerkens' hypothesis for central Nevada. That is, nonindependence of data is intrinsic to the hypothesis that point designs were copied from a single successful model through indirect bias. As Potter shows using simulations, nonindependent data of this kind result in artificially high correlations. Although Potteargues that these correlations are consequently "spurious," we argue that this tells us something extremely valuable: that indirect bias generates high interattribute correlations (spurious or not). Given that high correlations are what we found in Phase 1, we can extrapolate to Bettinger and Eerkens' (1999) archaeological data to infer that indirect bias was operating in central Nevada. 
Further, Potter's solution to what he sees as a problematic use of correlations misses the mark. For example, given that 66 of our participants in Phase 1 copied the same most-successful pretest participant, Potter states that "the 66 identical points provide no more information about the relationship of height to width than a single point" and suggests "treating the 66 points as a single point." We disagree. The fact that 66 of the points are identical tells us that they were all copied from the same person, and treating them all as a single point, while conventionally statistically correct, would overlook this crucial information.

Our response to Potter's second criticism is similar. He argues that because our participants are partitioned into different groups with different optimal point values, correlations cannot be used. Through simulations Potter shows that the size and significance of the correlations depend on the degree to which groups overlap in their designs: the more the groups overlap, the lower the overall interattribute correlations. Again, rather than this being "spurious," the result is exactly what we predicted. Indirectly biased cultural transmission generates low within-group variation (as group members all copy the same most-successful model in their group) and high betweengroup variation (assuming that the most-successful group member in each group has a different point design, which is expected given environmental differences or multiple locally stable optimal designs). This pattern constitutes nonoverlapping groups and thus high interattribute correlations. Guided variation generates high within- and between-group variation, as different hunters across all groups independently converge on different locally optimal designs without any within-group transmission. This would result in relatively more overlap between the groups and thus lower interattribute correlations. Potter's simulations, and our experimental findings, therefore confirm that interattribute correlations can be used to infer the operation of different learning biases, supporting Bettinger and Eerkens' (1999) hypothesis that high interattribute correlations in central Nevada resulted from indirect bias and low interattribute correlations in eastern California resulted from guided variation.

We fully acknowledge that our analyses violate conventional assumptions of correlation statistics. Yet when we know that a particular statistical pattern (high versus low interattribute correlations) is generated by a particular set of learning biases (indirect bias versus guided variation), we can use this information to explain observations in real archaeological data of the former patterns in terms of the latter learning biases, regardless of whether "conventional" statistical assumptions are violated.

Finally, we note that in our original study we also provided an alternative method of determining the population-level consequences of different learning processes that does not violate conventional statistical assumptions: comparing between- and within-group coefficients of variation. As already noted, indirect bias should reduce within-group cultural variation relative to between-group variation, whereas guided variation should not. Accordingly, we found significantly lower within-group variation in Phase 3 following the introduction of within-group cultural transmission than in Phase 2, during which cultural transmission was not permitted (e.g., see Mesoudi and O'Brien 2008a, Figure 2). See Eerkens and Lipo (2005) and Mesoudi and O'Brien (2008b) for more detailed simulations and analyses linking patterns of between- and within-group variation to different forms of cultural transmission.

In summary, we reiterate that rigorous and appropriate statistical methods are of the utmost necessity for testing hypotheses about culture change. That said, we stand by our experimental protocol, the methods we used, and most important, our conclusions. To our knowledge, Bettinger and Eerkens' work was the first significant archaeological study that examined cultural-transmission theory empirically. Similarly, our work is the first experimental study to bridge the gap between cultural-transmission theory and the empirical record. We hope it prompts further efforts. 


\section{References Cited}

Bettinger, Robert L., and Jelmer Eerkens

1999 Point Typologies, Culwras Transmission, and the Spread of Bow-and-Arrow Technology in the Prehistoric Great Basin. American Antiquity 64:231-242.

Boyd, Robert, and Peter J. Richerson

1985 Culture and the Evolutionary Process. University of Chicago Press, Chicago.

Eerkens, Jelmer W., and Carl P. Lipo

2005 Cultural Transmission, Copying Errors, and the Generation of Variation in Material Culture and the Archaeological Record. Journal of Anthropological Archaeology 24:316-334.
Mesoudi, Alex

2007 Using the Methods of Experimental Social Psychology to Study Cultural Evolution. Journal of Social, Evolutionary and Cultural Psychology 1:35-38.

Mesoudi, Alex, and Michael J. O'Brien

2008a The Cultural Transmission of Great Basin ProjectilePoint Technology I: An Experimental Simulation. American Antiquity 73:3-28.

2008b The Cultural Transmission of Great Basin ProjectilePoint Technology II: An Agent-Based Computer Simulation. American Antiquity 73:627-644.

Submitted November 10, 2010; Accepted November 10, 2010. 